\title{
An Innovative and Simple Impedance Matching Network Using Stacks of Metasurface Sheets to Suppress the Mismatch Between Antennas and RF Front-End Transceivers Circuits
}

\author{
Mohammad Alibakhshikenari ${ }^{1 *}$, Bal S. Virdee ${ }^{2}$, Ayman A. Althuwayb $^{3}$, Francisco Falcone ${ }^{4,5}$, and Ernesto \\ Limiti $^{1}$ \\ ${ }^{1}$ Electronic Engineering Department, University of Rome "Tor Vergata", Via del Politecnico 1, 00133 RM, Rome, Italy \\ ${ }^{2}$ London Metropolitan University, Center for Communications Technology, London N7 8DB, UK \\ ${ }^{3}$ Electrical Engineering Department, Jouf University, Sakaka, Aljouf 72388, Saudi Arabia \\ ${ }^{4}$ Electric, Electronic and Communication Engineering Department, Public University of Navarre, Pamplona, Spain \\ ${ }^{5}$ Institute of Smart Cities, Public University of Navarre, 31006 Pamplona, Spain \\ *alibakhshikenari@ing.uniroma2.it
}

\begin{abstract}
A innovative and simple impedance matching network is presented that is implemented by stacking together metasurface (MTS) sheets. The technique is shown to reduce the mismatch between free-space and $R F$ frontend antenna of a receiver. The MTS based impedance matching network is modeled as a transmission-line loaded with shunt and series capacitances and inductances, respectively. The proposed MTS impedance matching network can be employed to effectively interface the freespace to the antenna of an $\mathrm{RF}$ receiver and thereby optimize power absorption. Each MTS impedance matching sheet comprises two-dimensional periodic array of subwavelength microstrip resonator unit-cells that are spaced at a wavelength that is smaller than the frequency of operation. The unit-cells are square shaped patches and embedded with cross-shaped slots that are grounded through a via-hole. The MTS impedance matching network was fabricated using FR-4 substrate. 3D full-wave EM tool by Ansys HFSS ${ }^{\text {TM }}$ was used to verify its effectiveness. The proposed MTS impedance matching sheet is relatively easy to implement in practice.
\end{abstract}

Index Terms - Antenna impedance matching network, metasurface (MTS), RF front-end transceivers, 2-D array, microstrip resonators.

\section{INTRODUCTION}

Antenna is an essential component in a wireless communication system as it enables transmission or reception of RF signals in free-space [1-4]. The antenna should be impedance matched to the RF front-end of wireless systems as well as the free-space so that it operates optimally over its prescript frequency range in terms of radiation gain and efficiency [5]. Optimum efficiency will dictate the range of transmission, minimize energy consumption, reduce unwanted heating effects, and provide reliable data throughput [6]. The RF circuitry transfers the signal power via the $50 \Omega$ transmission-line to the antenna for transmission in free-space with impedance of $377 \Omega$, as illustrated in Fig.1.

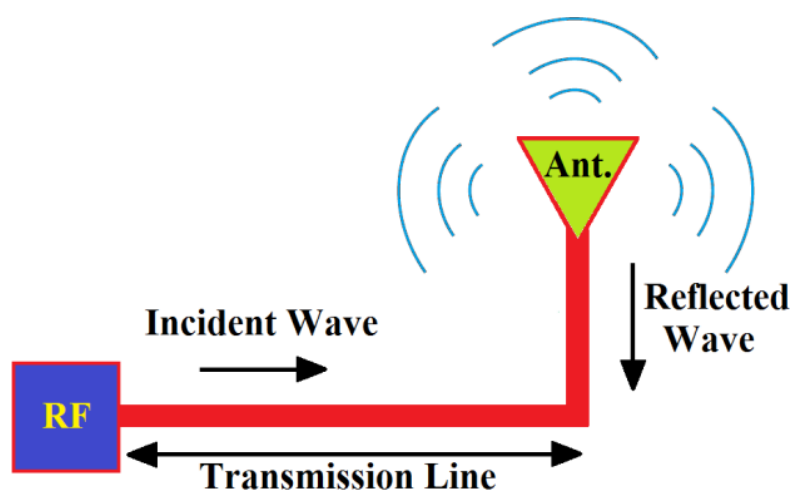

Fig.1. Antenna as an impedance transformer.

Matching the antenna's input impedance to $50 \mathrm{ohms}$ is a requirement to ensure optimal power is transferred from the RF circuitry to the antenna with negligible reflection $[7,8]$ as defined by the reflection-coefficient [9]:

$$
\Gamma=\frac{Z_{\text {antenna }}-Z_{R F \text { ciruit }}}{Z_{\text {antenna }}+Z_{R F \text { circuit }}}
$$

Impedance matching involves designing a network that transforms the input impedance of antenna $\left(Z_{\text {antenna }}\right)$ to the impedance of the RF front-end $\left(Z_{R F \text { circuit }}\right)$ that is typically 50 ohms. A perfect match is achieved when $Z_{\text {antenna }}=Z_{R F \text { circuit }}$. Under this condition, according to Equ.(1), $\Gamma=0$. If the impedance of the antenna and its feedline do not match, thus the impedance of the source is 
complex, which is dependent of the length of the line. The length of the feedline needs to be considered in the design as transforms the impedance of the antenna. Similarly, it's vital to match the antenna to free-space to optimize power transfer.

Proposed in the paper is a novel impedance matching technique to suppress the mismatch between the antenna of an RF receiver with free-space. This is achieved by stacking metasurface (MTS) sheets in front of the antenna. MTS is the 2-D equivalent of a metamaterial [10-13]. Implemented on the sheets are 2D array of the square patches with subwavelength periodicity. The MTS here was fabricated on the $0.6 \mathrm{~mm}$ FR-4 layer with $\varepsilon_{r}=4.3$, and $\tan \delta=0.025$. The MTS properties created by etching cross-shaped slots on the patches that are connecting to the ground-plane with via-holes.

\section{ANTENNA IMPEDANCE MAtChing Network BASED ON STACKIng Metasurface SHEETS}

In RF networks any mismatch in impedance leads power to be reflected from the impedance mismatch boundary to the RF source. This reflection generates undesired standing wave patterns that cause power storage instead of power transfer to the load. This results in diminished power flow to the load from the input or other segments of the RF system. The standing wave may cause other detrimental effects such as overheating the RF circuitry due to enhanced power level maxima along the transmission media. Benefits of proper impedance matching are reduction in amplitude and phase error, reduction in power loss and improvement in the signal to noise ratio.

The design of impedance matching networks is challenging, and in antennas the degree of matching will determine their performances such as return-loss, impedance bandwidth, radiation gain and efficiency. Typical antenna impedance consists of resistive and reactive components, hence the matching circuit required needs to be complex conjugate of this impedance for realizing optimum matching conditions.

EM-waves can be manipulated by metasurfaces which are artificially engineered two-dimensional subwavelength structures arranged in a periodic array. Unlike frequency selective surfaces (FSS) the spacing or periodicity of the resonators in metasurfaces is small compared to the wavelength at the frequency of interest [14-17]. EM-waves incident on the metasurface induce electric and magnetic dipole moments, which are related to the effective permittivity and permeability of the composite medium. When the individual structures are resonant, the overall metasurface structure exhibits negative values for both, relative permittivity and permeability. This happens because the phase response of the resonance reverses.
The basic MTS sheet shown in Fig.2(a) consists a 2-D periodic array of square microstrip patches or unit-cells implemented on FR-4 dielectric substrate. Shown in Fig.2(b) is the MTS sheet with cross-shaped slots embedded in each metallic patch that is linked to the ground-plane through a via-hole. The capacitance of patches coupled with the inductance of the vias create an $L C$ resonant structure with the resonant frequency much smaller than the wavelength at the operating frequency.

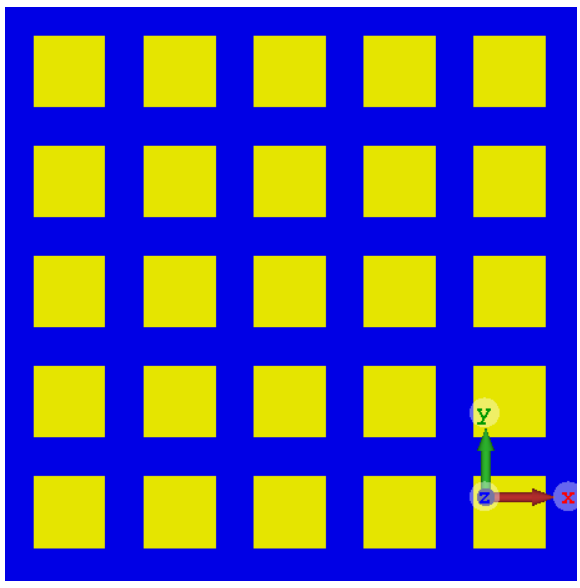

(a)

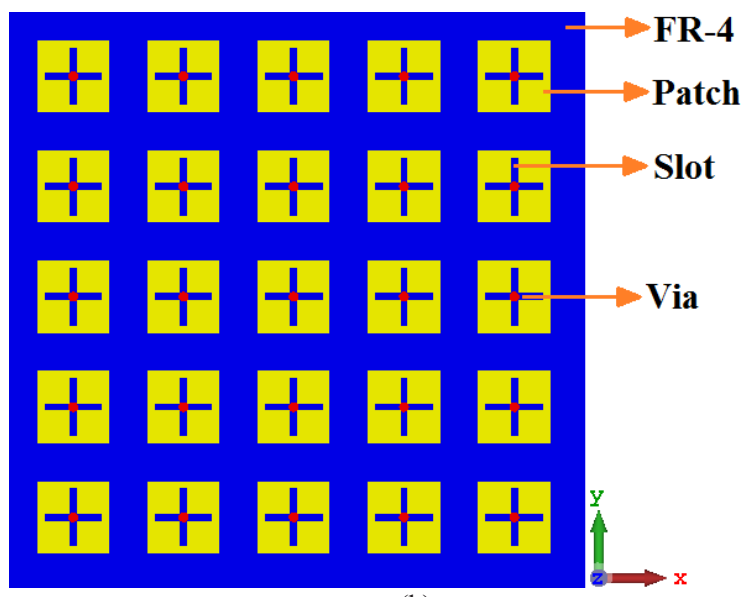

(b)

Fig.2. Metasurface sheet constructed from a 2-D periodic array of the square microstrip unit-cells, (a) basic structure without slot-line and via inclusions, and (b) with slot-line and via inclusions.

The top-layer patches are made with standard PCB manufacturing technology. The length of the slots etched are in the order of 0.026 of the wavelength of operation. The gap between the patches is also in the order of 0.026 of the wavelength of operation. The symmetric design offers polarization insensitivity property. This structure prevents unwanted currents to flows on the MTS surface therefore suppressing undesired mutual coupling which can adversely affect its characteristics [10]. It can be shown that when the array is exposed to EM-waves the 
magnitudes of the effective electric and magnetic polarizations are identical [18]. This means that the MTS sheets absorbs the EM-waves without generating any backward wave, i.e., reflection.

Structural parameters of the proposed MTS sheet are listed in Table I. The proposed MTS sheet is easy to fabricate for operation at microwave frequencies however requires high precision laser cutting at millimeter-wave frequencies. The MTS sheet can be used for impedance matching of antennas of the RF front-end of wireless systems to free-space medium.

TABLE I. STRUCTURAL PARAMETERS (Units in millimeter).

\begin{tabular}{|c|c|}
\hline Length / Width of the substrate & $40 / 40$ \\
\hline Substrate thickness & 0.8 \\
\hline Length / Width of the patches & $5 / 5$ \\
\hline Length / Width of the slots & $2 / 0.5$ \\
\hline Gap between the patches & 2 \\
\hline Height / Diameter of the via & $0.8 / 0.35$ \\
\hline
\end{tabular}

The proposed MTS sheet is used as an impedance matching shield which is unlike traditional transmissionline or lumped element matching networks [19-22]. By stacking together MTS sheets, as illustrated in Fig.3, the incident of EM-waves induces electric and magnetic polarizations in each sheet that strongly absorb the waves without any unwanted reflection [23]. The MTS unit-cells can be modeled using transmission-line theory in terms of series and shunt $L C$ components, as in Fig.3(c). It can be shown that the MTS exhibits left-handed properties [24]. Magnitude of the $L C$ parameters, which were obtained from Ansys HFSS ${ }^{\text {тм }}$ full-wave EM simulation, are given in Table II.

TABLE II. EXTRACTED CIRCUIT PARAMETERS FOR EACH METASURFACE SHEET.

\begin{tabular}{|c|c|c|c|}
\hline$C_{L}$ & $L_{L}$ & $C_{R}$ & $L_{R}$ \\
\hline $1.85 \mathrm{pF}$ & $2.10 \mathrm{nH}$ & $0.85 \mathrm{pF}$ & $1.25 \mathrm{nH}$ \\
\hline
\end{tabular}

The simulated performance parameters of the 2-D periodic array of stacked non-MTS and the MTS sheets are shown in Fig.4. The results show the significantly improved performance of the stacked MTS sheets in terms of return-loss, impedance bandwidth, radiation gain, and radiation efficiency. The average improvement in return-loss is $-10 \mathrm{~dB}$, gain is $2 \mathrm{dBi}$, and efficiency is $20 \%$ across the frequency range of $3.0-5.0 \mathrm{GHz}$. These results verify the feasibility of the proposed MTS for impedance matching an antenna with an RF front-end of wireless systems.
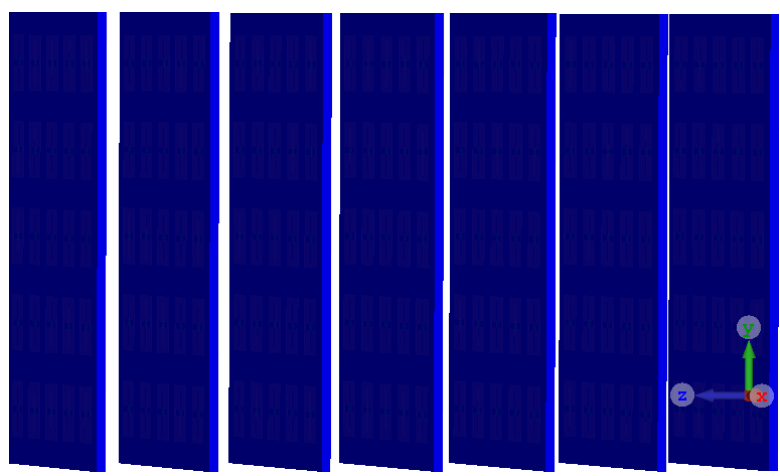

(a)

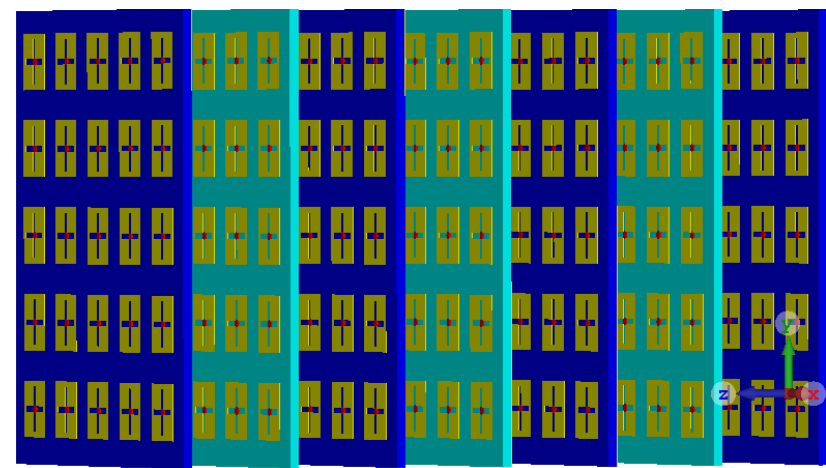

(b)

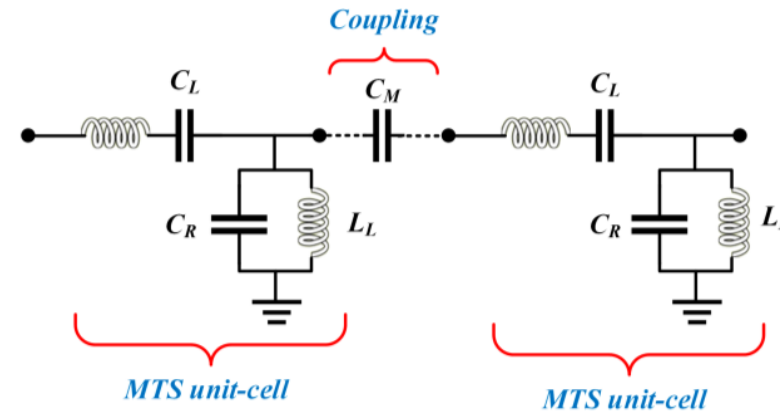

(c)

Fig.3. (a) Stack of seven non-MTS sheets, (b) MTS impedance matching sheets, and (c) Equivalent circuit model of unit-cells.

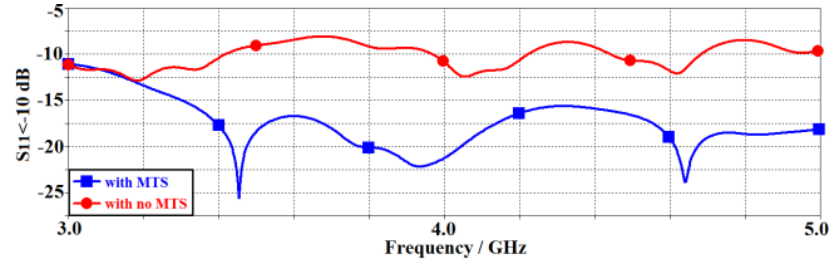

(a) 


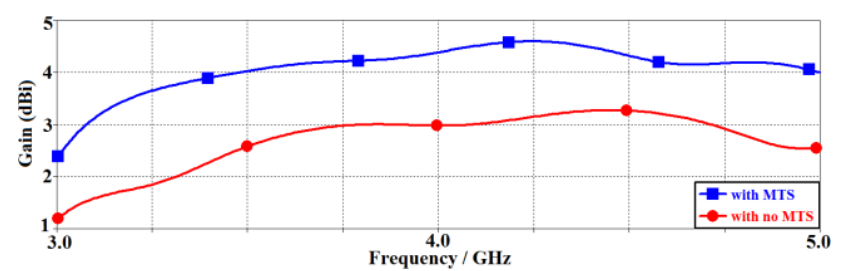

(b)

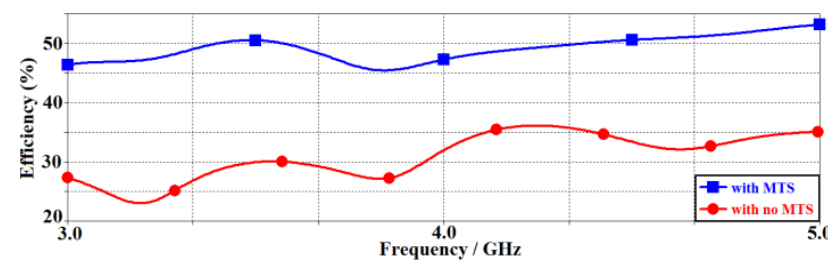

(c)

Fig.4. Simulated performance parameters of the 2-D periodic array of stacked non-metasurface sheets and metasurface sheets, (a), return-loss response, (b) radiation gain, and (c) radiation efficiency.

\section{CONCLUSIONS}

Stack of MTS sheets are shown to effectively transform the impedance of free-space to match with the impedance of RF receiver. The MTS sheet is implemented with a periodic arrangement of square microstrip patches in which are etched cross-shaped slots that is linked to the ground-plane through a via-hole. The gap between the patches is subwavelength at the desired operating wavelength. The capacitance created by the patches combined with the inductance of the vias create an $L C$ resonant structure with the resonant frequency much smaller than the wavelength at the operating frequency. As a result, the proposed structure is shown to absorb any incident RF-energy with no reflection. The proposed impedance matching technique is shown to significantly enhance the antenna's radiation gain and efficiency characteristics.

\section{ACKNOWLEDGMENTS}

This work is partially supported by RTI2018-095499B-C31, Funded by Ministerio de Ciencia, Innovación y Universidades, Gobierno de España (MCIU/AEI/FEDER,UE).

\section{REFERENCES}

[1] Constantine A. Balanis, "Modern Antenna Handbook", ISBN: 978-0470-03634-1 September 2008, 1712 Pages, John Wiley \& Sons, Inc.

[2] Constantine A. Balanis, "Antenna Theory: Analysis and Design", 4th Edition, ISBN: 978-1-118-64206-1, February 2016, 1104 Pages.

[3] William A. Imbriale; Steven (Shichang) Gao; Luigi Boccia, "Antenna Basics," in Space Antenna Handbook, Wiley, 2012, pp.1-35, doi: 10.1002/9781119945147.ch1.

[4] Alejandro Aragon-Zavala, "Antennas," Indoor Wireless Communications: From Theory to Implementation, Wiley, 2017 , pp.167-214, doi: 10.1002/9781119004547.ch6.

[5] M. Alibakhshikenari, et al., "Impedance Matching Network Based on Metasurface (2-D Metamaterials) for Electrically Small Antennas", 2020
IEEE International Symposium on Antennas and Propagation and North American Radio Science Meeting (2020 IEEE AP-S/URSI), pp.19531954, Montréal, Québec, Canada on 5-10 July 2020.

[6] M. Alibakhshikenari, et al., "Automated Reconfigurable Antenna Impedance for Optimum Power Transfer," 2019 IEEE Asia-Pacific Microwave Conference (APMC), Singapore, 2019, pp. 1461-1463.

[7] A. A. G. Amer, S. Z. Sapuan, N. Nasimuddin, A. Alphones and N. B. Zinal, "A Comprehensive Review of Metasurface Structures Suitable for RF Energy Harvesting," in IEEE Access, vol. 8, pp. 76433-76452, 2020, doi: 10.1109/ACCESS.2020.2989516.

[8] M. Alibakhshikenari, et al., "Improved Adaptive Impedance Matching for RF Front-End Systems of Wireless Transceivers", Scientific Reports, 10, 14065, 2020.

[9] Anitha Govind, "Antenna Impedance Matching - Simplified", Abracon LLC, pp.1-6.

[10] Douglas H. Werner, "Broadband Metamaterials in Electromagnetics, Technology and Application", ISBN 9789814745680 , July 6, 2017, Jenny Stanford Publishing, 398 Pages.

[11] C. L. Holloway, E. F. Kuester, J. A. Gordon, J. O'Hara, J. Booth and D. R. Smith, "An Overview of the Theory and Applications of Metasurfaces: The Two-Dimensional Equivalents of Metamaterials," IEEE Antennas and Propagation Magazine, vol. 54, no. 2, pp. 10-35, April 2012, doi: 10.1109/MAP.2012.6230714.

[12] Badawe, M., Almoneef, T. \& Ramahi, O. "A True Metasurface Antenna," Sci Rep 6, 19268 (2016). https://doi.org/10.1038/srep19268.

[13] Aobo Li, Shreya Singh, and Dan Sievenpiper, "Metasurfaces and their applications," Nanophotonics, Volume 7, Issue 6, Pages 989-1011.

[14] Karim Achouri, et al., "Dielectric Resonator Metasurface for Dispersion Engineering," IEEE Transactions on Antennas and Propagations, vol. 65, no. 2, pp. 673-680, February 2017.

[15] Kim, M., Eleftheriades, G.V., "Design and Demonstration of Impedance-matched Dual-band Chiral Metasurfaces," Sci Rep 8, 3449 (2018).

[16] M. Alibakhshikenari, et al., "Metasurface for Controlling Polarization of Scattered EM Waves," 2020 4th Australian Microwave Symposium (AMS), Sydney, Australia, February 2020, pp. 1-2.

[17] A. Arbabi, et al., "Dielectric metasurfaces for complete control of phase and polarization with subwavelength spatial resolution and high transmission," Nature Nanotechnol., vol. 10, pp. 937-943, Aug. 2015.

[18] R. Alaee, M. Albooyeh and C. Rockstuhl, "Theory of metasurface based perfect absorbers," Journal of Physics D: Applied Physics, vol. 50, 2017, pp. 1-14.

[19] C. Ko and G. M. Rebeiz, "A 1.4-2.3-GHz Tunable Diplexer Based on Reconfigurable Matching Networks," IEEE Transactions on Microwave Theory and Techniques, vol. 63, no. 5, pp. 1595-1602, 2015. [20] M. Gilasgar, et al., "A $2.4 \mathrm{GHz}$ CMOS Class-F Power Amplifier with Reconfigurable Load-Impedance Matching," IEEE Transactions on Circuits and Systems I: Regular Papers, vol.66, no.1, pp.31-42, 2019.

[21] Y. Yoon, et al., "A 2.4-GHz CMOS Power Amplifier with an Integrated Antenna Impedance Mismatch Correction System," in IEEE Journal of Solid-State Circuits, vol. 49, no. 3, pp. 608-621, March 2014. [22] Z. Hameed, K. Moez, "Design of Impedance Matching Circuits for RF Energy Harvesting Systems," Microelectronics Journal, vol. 62, 2017, pp. 49-56.

[23] Y. Yang, et al., "All-Dielectric Metasurface Analogue of Electromagnetically Induced Transparency," Nature Commun., vol. 5, no. 5753, pp. 366-368, 2014.

[24] Christophe Caloz, Tatsuo Itoh, Electromagnetic Metamaterials: Transmission Line Theory and Microwave Applications, Wiley-IEEE Press, Dec. 2005. 